\title{
Two-Photon Microscopy of TLR4 Expressing Cells in Intact Mouse Kidney
}

\author{
Guixue Bu, Jeremy Wellen and PandurangaRao Varada \\ Worldwide Research \& Development, Pfizer Inc., Andover, Massachusetts, USA
}

Two-photon excitation fluorescence (TPEF) microscopy is proven to be a powerful tool in biomedical research by examining exogenous and/or endogenous fluorescent molecules [1]. In particular, many biological tissues have endogenous fluorescent molecules which are excitable with infrared laser in the 700 to $750 \mathrm{~nm}$ range in TPEF microscopy [2], offering a unique opportunity to visualize unprocessed fresh tissues. It is now feasible to image both endogenous and exogenous fluorophores with a tunable laser by quickly switching between the optimal excitation wavelengths or with synchronized pulses from two lasers [3]. In this study, we investigated the autofluorescence of the kidney and located the image toll-like receptor 4 (TLR4) expressing cells in the renal cortex, a potential therapeutic target for acute kidney injury, using sequential TPEF microscopy and a mouse model of acute, low-dose lipopolysaccharides (LPS, a specific TLR4 ligand) challenge.

Materials and Methods

Qtracker ${ }^{\circledR} 565$ non-targeted quantum dots (QDs) and Alexa 488-labeled LPS from e-coli (Alexa488) were purchased from Invitrogen (Eugene, OR). All animal protocols were approved by Pfizer Institutional Animal Care and Use. Male C57BL/6J mice about 10 weeks old and 20-30 grams were obtained from The Jackson Laboratory (Bar Harbor, ME). Alexa488 (30 $\mu \mathrm{g}$ in $100 \mu \mathrm{L}$ PBS) and QDs (30 $\mu \mathrm{L}$ stock solution in $70 \mu \mathrm{L}$ PBS) were administered intravenously 90 min and 10 min, respectively, prior to carbon dioxide asphyxiation euthanasia. Kidneys were also harvested from naïve mice. The intact kidneys were kept in chilled PBS until imaging on a multiphoton microscope equipped with a Chameleon Vision infrared laser tuneable from 680 to $1080 \mathrm{~nm}$ (Leica TCS SP5 MP, Leica Microsystems, Inc., Chicago, IL). A Leica 20×, 1.0 NA water immersion objective and two nondescanned detectors (NDDs) with a FITC/TRITC filter cube were used. Each kidney was immersed and immobilized with a custom-made tissue anchor in a glass Petri dish filled with PBS. Images were sequentially obtained with $860 \mathrm{~nm}$ excitation for administrated probes and $740 \mathrm{~nm}$ excitation for tissue autofluorescence, respectively. Emission spectra with $20 \mathrm{~nm}$ bandwidth and $10 \mathrm{~nm}$ step were acquired using the spectrophotometer detector of the microscope in the xy $\lambda$ mode. All images were acquired with 16-bit resolution. Emission spectra were calculated with custom software written in Matlab (The MathWorks, Natick, MA).

\section{Results}

Tubular Structures of Renal Cortex by Autofluorescence. Kidneys from naïve mice were imaged with various excitation wavelengths ranging from 720 to $820 \mathrm{~nm}$. The signal intensity reached the highest at $740 \mathrm{~nm}$ excitation. As shown in Fig. 1A., the tubular structures delineated by the autofluorescence are similar to observations in histological studies or in vivo two-photon microscopy of renal cortex [4]. The autofluorescence intensity appears inhomogeneous across the field of view with most tubules are bright but some are dim. Fig. 1B shows an image from a representative xy $\lambda$ scan. Polygon ROIs were drawn on both bright and dim tubules. Fig. 1C shows the representative normalized emission spectra calculated for two ROIs in Fig. 1B, one on a bright tubule (red ROI) and another on a dim tubule (green ROI). There is only slight difference between the two spectra, suggesting the autofluorescence be from the same fluorescent molecules and the inhomogeneity in autofluorescence intensity be caused by the 
difference in the concentration of the molecules. The spectra have similar profile as NADH solution [2], suggesting NADH is the primary source of the autofluorescence in the ex vivo mouse renal cortex.

TLR4 Expressing Cells Mainly at the Brush Border of the Proximal Tubules (PTs). Fig. 2 shows a representative image of an intact kidney harvested from a mouse administrated with Alexa488 and QDs. Fig. 2A is the autofluorescence for structural information of the optical section. There is no obvious distortion to the tubules caused by the administrated LPS and QDs. The Alexa488 shown in Fig. 2B was found mainly in the tubules, aligning with the luminal border of the tubules. The morphology and the brightness of autofluorescence suggest that these tubules are PTs. This observation is consistent with previous report [4]. In contrast, the peritubular perfusion is illuminated by the non-targeted QDs, as in Fig. 2C. The non-targeted QDs cannot pass the glomerular filtration and are not expected in the tubules after the glomeruli, as confirmed by the merged image in Fig. 2D. The QDs appeared only on the basolateral side of the tubules, indicating the QDs remain in the blood of peritubular perfusion.

\section{Conclusions}

We located the TLR4 expressing cells in intact mouse kidneys using sequential two-photon multicolor imaging of the endogenous molecules and administrated probes. Such strategy can be applied to a variety of studies such as biodistribution and target identification in the early stage of drug discovery.

\section{References:}

[1] WR Zipfel et al, Nat. Biotechnol. 21 (2003), p.1369.

[2] WR Zipfel et al, Proc. Natl, Acad. Sci. 100 (2003), p.7075.

[3] P Mahou et al, Nat. Methods 9 (2012), p.815.

[4] R Kalakeche et al, J. Am. Soc. Nephrol. 22 (2011), p.1505.

A

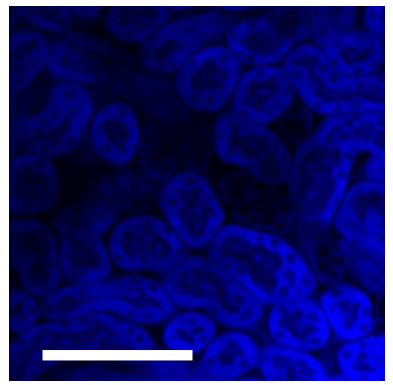

B

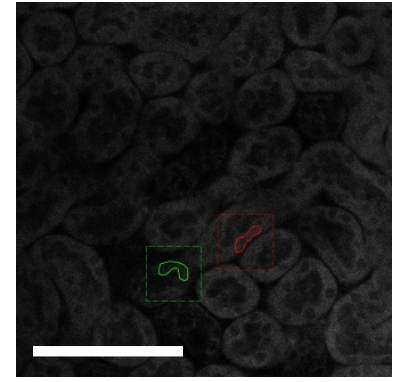

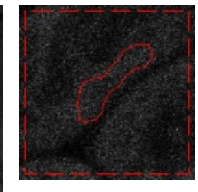

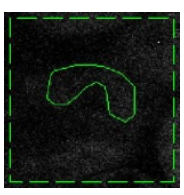

C

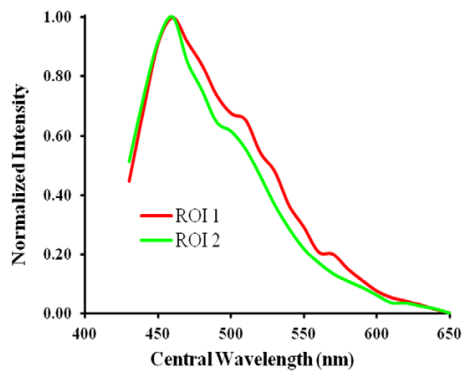

Figure 1. Autofluorescence of mouse renal cortex with $740 \mathrm{~nm}$ excitation. (A) A representative optical section of renal cortex with DAPI filter. (B) An image from a xy $\lambda$ scan with two polygon ROIs on a bright and a dim tubules. (C) Emission spectra of the two ROIs in (B). Scale bars: $100 \mu \mathrm{m}$.
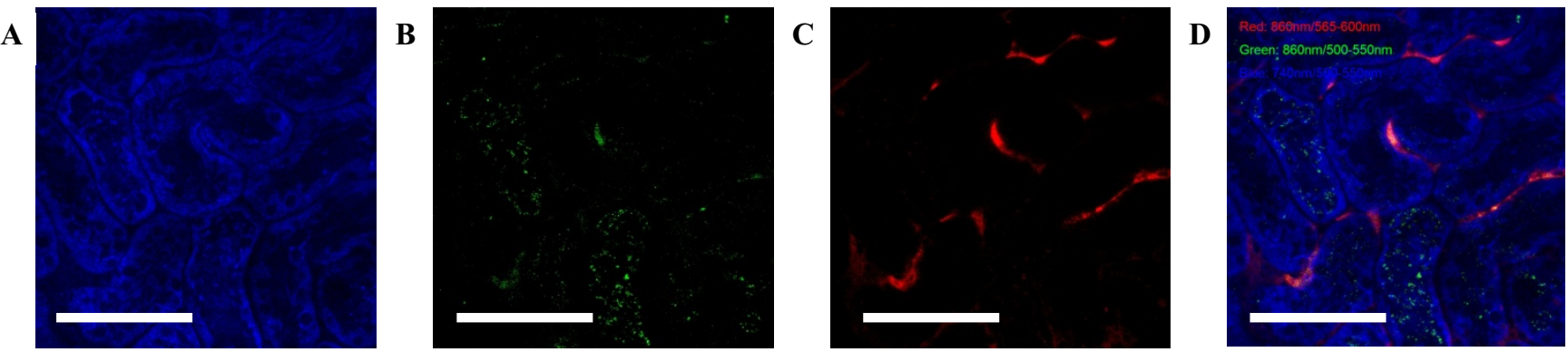

Figure 2. Multicolor image of mouse renal cortex with sequential 860 and $740 \mathrm{~nm}$ excitation. (A) Autofluorescence with $740 \mathrm{~nm}$ excitation. (B) and (C) Alexa488 and QD fluorescence with $860 \mathrm{~nm}$ excitation. (D) Merged image of (A), (B) and (C). Scale bars: $100 \mu \mathrm{m}$. 